\title{
Sintering temperature dependence of evolving morphologies and magnetic properties of Ni0.5Zn0.5Fe2O4 synthesized via mechanical alloying
}

\begin{abstract}
We report on an investigation to unravel morphological and magnetic-property dependence

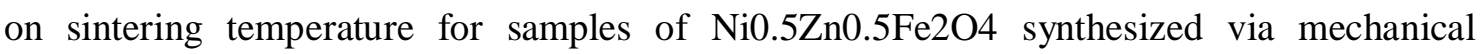
alloying. The samples were sintered at various sintering temperatures from $800{ }^{\circ} \mathrm{C}$ to 1000 ${ }^{\circ} \mathrm{C}$. The morphology of the samples was studied by means of scanning electron microscopy (SEM); hysteresis and permeability measurement were carried out using a $\mathrm{BH}$ hysteresisgraph system and an impedance analyzer, respectively. The morphological studies show a microstructural evolution with the increase of sintering temperature. The relationship between ordered magnetism and the microstructure of the samples show that the important grain-size threshold for the appearance of significant ordered magnetism (mainly ferromagnetism) is about Ó.3 $\mathrm{\varepsilon m}$. We found that two factors sensitively influenced the samples content of ordered magnetism, their ferrite-phase crystallinity degree and the number of grains above the critical grain size.
\end{abstract}

Keyword: Hysteresis; Magnetic materials; Magnetic properties; Sintering 